Breast Cancer

\title{
A Prospective Study of Taxane-Induced Neuropathy with Breast Cancer: Proper Assessment Tool for Taxane-Induced Neuropathy
}

\author{
Eun Hee Sohn ${ }^{1}$ Jin Sun Lee ${ }^{2}$ Mi Sook Jung ${ }^{3}$ Je Ryong Kim²
}

${ }^{1}$ Department of Neurology, Chungnam National University Hospital, Korea

2Department of Surgery and Research Institute for Medical Sciences, College of Medicine, Chungnam National University, Korea

${ }^{3}$ College of Nursing, Chungnam National University, Daejeon, Korea
Address for correspondence Eun Hee Sohn, MD, PhD, Department of Neurology, Chunanam National University Hospital, 282 Moonwha-ro, Jung-gu, Daejeon, South Korea 35015, (e-mail: seh337@daum.net).

South Asian J Cancer 2021;10:58-63.

\section{Abstract}

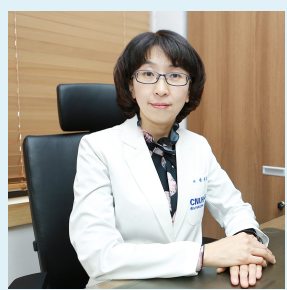

Eun Hee Sohn

\section{Keywords}

- breast neoplasms

- taxane

- peripheral nervous system diseases

- small fiber neuropathy
Background Many chemotherapeutic agents, especially taxanes, can induce peripheral neuropathy.

Aim To evaluate the clinical characteristics of taxane-induced neuropathy (TIN) and determine the proper assessment tool for TIN in patients with breast cancer.

Setting and Design Single-center, observational, prospective study.

Methods and Material Forty-three patients with breast cancer treated with taxanes were prospectively enrolled. The reduced version of the Total Neuropathy Score (TNSr) was performed at baseline and 3 months after enrollment. TIN was diagnosed if the difference between the baseline and 3-month TNSr was greater than 1. In patients with TIN, the European Organization for Research and Treatment of Cancer Quality of Life Questionnaire- Chemotherapy-Induced Peripheral Neuropathy (20-item scale (EORTC-CIPN20) was also assessed 3 months after enrollment.

Results Thirty-seven out of $43(86.0 \%)$ patients were diagnosed with TIN. Sensory symptoms (64.9\%) were the most frequent abnormality, followed by autonomic symptoms (54.1\%). No patients reported motor symptoms or motor weakness. The TNSr sensory symptom score positively correlated with that of the EORTC-CIPN20. Nerve conduction studies showed reduced nerve conduction velocities and amplitudes after taxane treatment compared to those before chemotherapy in all tested nerves; however, only three $(8.1 \%)$ patients had sural sensory nerve action potential amplitude outside normal limits.

Conclusions TIN was predominantly sensory with normal nerve conduction studies which is the main feature of small fiber neuropathy. A combination scale comprising of a clinician-based scale and a patient-reported questionnaire and specialized tests for small nerve fibers should be considered as proper assessment tools to evaluate TIN.
How to cite this article: Sohn E. H, Lee J. S, Jung M. S, Kim J. R. A Prospective Study of Taxane-Induced Neuropathy with Breast Cancer: Proper Assessment Tool for Taxane-Induced Neuropathy. South Asian J Cancer 2021;10(2):58-63.
(C)2021. MedIntel Services Pvt Ltd.

This is an open access article published by Thieme under the terms of the Creative Commons Attribution-NonDerivative-NonCommercial-License, permitting copying and reproduction so long as the original work is given appropriate credit. Contents may not be used for commercial purposes, or adapted, remixed, transformed or built upon. (https://creativecommons.org/licenses/by-nc-nd/4.0/).

Thieme Medical and Scientific Publishers Private Ltd A-12, Second Floor, Sector -2, NOIDA -201301, India 


\section{Introduction}

Chemotherapy-induced peripheral neuropathy (CIPN) typically presents as length-dependent axonopathy and is one of the major dose-limiting side effects of chemotherapeutic agents. Many chemotherapeutic agents, especially taxanes (e.g., paclitaxel, docetaxel), platinum compounds (e.g., cisplatin, carboplatin, oxaliplatin), thalidomide, and bortezomib, can cause CIPN. Taxanes are microtubule-stabilizing drugs and are used to treat various malignancies, including breast, ovarian, and non-small cell lung cancers. ${ }^{1}$ Taxane-induced neuropathy (TIN) typically presents as sensory neuropathy, and motor impairment is rare ${ }^{2}$; however, a recent study observed a considerable prevalence of motor impairment in TIN. ${ }^{3}$ In that study, they used a clinician-based scale-the National Cancer Institute Common Terminology Criteria for Adverse Events (NCI-CTCAE)-that often overestimates motor neuropathy and could be a confounding factor. However, little attention has been given to motor impairments in CIPN; therefore, they recommended a more detailed assessment of motor symptoms when evaluating CIPN.

The prevalence of CIPN is highly variable and ranges from 12.1 to $96.2 \%{ }^{4}$ The variability in the prevalence may originate from heterogeneity in cancer or chemotherapeutic agents, assessment tools, or study design. There is currently no gold standard for the assessment of CIPN, but several clinician-based grading scales and patient-reported questionnaires have been developed. The NCI-CTCAE is the most commonly used clinician-based grading scale for $\mathrm{CIPN}^{5}$; however, it demonstrated high intraobserver disagreement and interobserver variability as well as overestimation of CIPN. ${ }^{6,7}$ Although many studies have used the NCI-CTCAE to identify CIPN, it is not a reliable assessment tool for clinical research. ${ }^{7}$ The European Organization for Research and Treatment of Cancer Quality of Life Questionnaire-CIPN 20-item scale (EORTC-CIPN20) is a patient-reported outcome measure, ${ }^{8}$ and its validity in CIPN has been demonstrated by several studies. ${ }^{7,9}$ However, patient-reported symptom questionnaires have not been routinely integrated as the sole method to identify CIPN, potentially due to feasibility concerns and the lack of consensus regarding the best available patient-based CIPN assessment method. ${ }^{2}$ The combination of a patient-reported outcome measure and a clinician-rated scale is regarded as a reasonable and practical method to determine CIPN. The Total Neuropathy Score (TNS), which was developed and validated for diabetic neuropathy, is a mixed scale of patient-reported symptoms and clinician-detected signs, including a physical examination for motor and sensory function with neurophysiological studies. ${ }^{10}$ The TNS and its reduced version (TNSr) have been used for CIPN assessment and have shown higher sensitivity and lower interobserver variability than those of other clinician-administered scales. ${ }^{11,12}$

For this study, we enrolled patients with breast cancer who were treated with taxanes to reduce the heterogeneity of subjects. We used serial TNSr, assessed before and after chemotherapy, for a more precise evaluation of motor function and a more accurate diagnosis of CIPN. This study aimed to evaluate the clinical characteristics of TIN and the use of longitudinal TNSr for the diagnosis of TIN.

Table 1 Comparison of clinical features according to taxane-induced neuropathy status

\begin{tabular}{|c|c|c|c|}
\hline Variables & No TIN $(n=6)$ & $\operatorname{TIN}(n=37)$ & $p$-Value \\
\hline Age $($ mean $\pm S D)$ & $49.7 \pm 4.5$ & $48.9 \pm 10.4$ & ns \\
\hline Women (\%) & $6(100)$ & $37(100)$ & ns \\
\hline $\mathrm{BMI}\left(\mathrm{kg} / \mathrm{m}^{2}\right.$, mean $\left.\pm \mathrm{SD}\right)$ & $24.2 \pm 2.5$ & $23.9 \pm 3.6$ & ns \\
\hline $\begin{array}{l}<25(\%) \\
\geq 25(\%)\end{array}$ & $\begin{array}{l}4(66.7) \\
2(33.3)\end{array}$ & $\begin{array}{l}22(59.5) \\
15(40.5)\end{array}$ & ns \\
\hline $\begin{array}{l}\text { Cancer stage: I-III (\%) } \\
\text { IV (\%) }\end{array}$ & $\begin{array}{l}5(83.3) \\
1(16.7)\end{array}$ & $\begin{array}{l}35(94.6) \\
2(5.4)\end{array}$ & ns \\
\hline \multicolumn{4}{|l|}{ Taxane received } \\
\hline $\begin{array}{l}\text { Docetaxel (\%) } \\
\text { Paclitaxel (\%) }\end{array}$ & $\begin{array}{l}5(83.3) \\
1(16.7)\end{array}$ & $\begin{array}{l}30(81.1) \\
7(18.9)\end{array}$ & ns \\
\hline Chemotherapeutic regimen (\%) & & & ns \\
\hline AC with weekly taxane & $1(16.7)$ & $1(2.7)$ & \\
\hline AC with once every 3 week taxane & $0(0.0)$ & $16(43.2)$ & \\
\hline TC & $2(33.3)$ & $16(43.2)$ & \\
\hline TAC & $2(33.3)$ & $3(8.1)$ & \\
\hline TA & $1(16.7)$ & $1(2.7)$ & \\
\hline Cumulative dose & & & ns \\
\hline $\begin{array}{l}\text { Docetaxel (mean } \pm \text { SD) } \\
\text { Paclitaxel }(\text { mean } \pm \text { SD })\end{array}$ & $\begin{array}{l}637.8 \pm 151.2 \\
1799.2\end{array}$ & $\begin{array}{l}543.6 \pm 266.1 \\
1039.7 \pm 413.2\end{array}$ & \\
\hline
\end{tabular}

Abbreviations: AC, adriamycin and cyclophosphamide; ns, not significant; TA, taxane and adriamycin; TAC, taxane, adriamycin, and cyclophosphamide; TC, taxane and cyclophosphamide; TIN, taxane-induced neuropathy. 


\section{Materials and Methods}

\section{Study Design}

This was a single-center, observational, prospective study.

\section{Participants}

We prospectively enrolled patients with breast cancer who received chemotherapy with docetaxel or paclitaxel. All enrolled patients were older than 20 years and received more than four cycles of combination of adriamycin and/or cyclophosphamide and/or taxane and 18 patients followed by additional taxane weekly or once every 3 weeks ( - Table 1 ). Patients were excluded if they had pre-existing risk factors for neuropathy such as diabetes mellitus, thyroid disease, other potential neurotoxic medication history, alcohol, or family history of neuropathy. We also excluded patients who could not give informed consent for any reason. This study was approved by the Chungnam National University ethics committee and conformed to the Declaration of Helsinki and its amendments.

\section{Clinical Assessment and the Definition of TIN}

The TNSr was used to diagnose TIN. The TNS, a clinician-based scoring system, is composed of subjective symptoms (e.g., sensory, motor, and autonomic symptoms), physical examination (pinprick and vibration sensation assessment, strength, and tendon reflex), and neurophysiological tests (e.g., nerve conduction studies [NCS] and vibration sense assessment by quantitative sensory testing [QST]), scored from 0 to 4 for each item. The total score ranges from 0 to 40 , and a higher score indicates more severe neuropathy. The TNSr is a reduced version of the TNS that eliminates the QST for vibration sensation but retains the NCS and clinical assessment. The TNSr was assessed at baseline (TNSr_B) and 3 months after enrollment (TNSr_F). TIN was diagnosed if the difference between the TNSr_F and TNSr_B was greater than 1 . The patients were divided into TIN and no TIN groups according to their diagnosis.

The EORTC-CIPN20 was administered to the TIN group 3 months after enrollment (CIPN20_F). The EORTC-CIPN20 is a 20-item self-reported questionnaire and has three subscales containing 9 items for sensory symptoms, 8 items for motor symptoms, and 3 items for autonomic symptoms. Each item is scored from 1 to 4 , and the total score ranges from 20 to 80 , with higher scores indicating worse symptoms. The CIPN20_F was compared with the TNSr_F in the TIN group.

We further evaluated body mass index, cancer stage, chemotherapy interval (weekly vs. once every 3 weeks), and cumulative doses. These clinical data were compared between the TIN and no TIN groups.

\section{Neurophysiological Assessment}

Sensory NCS were conducted on the median, ulnar, and sural sensory nerves and motor NCS were performed on the median, ulnar, and peroneal motor nerves. The nerve conduction velocities (NCV) and amplitudes of each nerve before and after chemotherapy were compared in the TIN group.

\section{Statistical Analysis}

Descriptive statistics were used to demonstrate the characteristics of the data. Continuous variables were analyzed using the Mann-Whitney U test, and categorical data were analyzed using Pearson's nonparametric chi-square test. We used Spearman correlations to evaluate the association between TNSr_F and CIPN20_F. All of the analyses were performed using SPSS (IBM Corp., released 2016, IBM SPSS Statistics for Windows, Version 24.0, Armonk, New York).

\section{Results}

In total, 43 female patients were enrolled (age [mean \pm SD] $49.0 \pm 9.8$ years; range, $20-67$ years). Thirty-seven out of 43 (86.0\%) patients were diagnosed with TIN. There were no significant differences in clinical features between the TIN and no TIN groups ( - Table 1). Neither chemotherapy interval (weekly vs. once every 3 week) nor cumulative doses of taxane showed differences between TIN and no TIN group (-Table 1).

Nine patients (20.9\%) had abnormal symptoms and/or physical examinations before chemotherapy, as evaluated by the TNSr_B; two patients had sensory symptoms, four patients had autonomic symptoms, five patients had an

Table 2 Patients with symptoms and abnormal physical examination at baseline and possible underlying disease in each patient

\begin{tabular}{|l|l|l|l|l|l|}
\hline \multirow{2}{*}{ Patient no. } & \multicolumn{2}{|c|}{ Symptoms } & \multicolumn{2}{c|}{ Physical examination abnormality } & \multirow{2}{*}{ Underlying disease } \\
\cline { 2 - 6 } & Sensory & ANS & Vibration & Tendon reflex & \\
\hline 1 & + & + & + & + & Radiculopathy \\
\hline 2 & - & - & - & + & Radiculopathy \\
\hline 3 & - & - & + & - & Radiculopathy \\
\hline 4 & + & + & + & + & None \\
\hline 5 & - & + & - & - & None \\
\hline 6 & - & + & - & - & None \\
\hline 7 & - & - & - & + & None \\
\hline 8 & - & - & - & + & None \\
\hline 9 & - & + & - & - & None \\
\hline
\end{tabular}

Abbreviations: ANS, autonomic nervous system; no., number. 
abnormal tendon reflex, and three patients had abnormal vibration sensation. Three patients had underlying radiculopathy, and the remaining six patients denied any previous diseases ( $\rightarrow$ Table 2). The changes in TNSr after chemotherapy were the largest in sensory symptoms, followed by autonomic symptoms, abnormal vibration sensation, and tendon reflex ( - Fig. 1 ). No patients reported motor symptoms or motor weakness.

In the TIN group, 24 out of 37 (64.9\%) patients reported sensory symptoms, and 20 patients (54.1\%) experienced more than one autonomic symptom according to the TNSr_F. Eighteen out of 25 (72.0\%) patients reported hand sensory symptoms (e.g., tingling, burning, or prickling pain), and 17 patients $(68.0 \%)$ reported autonomic symptoms as assessed by the CIPN20_F. The CIPN20_F sensory symptom scores were significantly correlated with the TNSr_F sensory symptom and pin sensation scores ( - Table 3 ).

The NCV and amplitudes of all tested nerves were significantly reduced following chemotherapy compared to those before chemotherapy (-Table 4). However, the postchemotherapy NCV and amplitudes were mostly within the normal range; only three patients $(8.1 \%)$ had sural sensory nerve action potential amplitudes outside the normal range.

\section{Discussion}

In this study, TIN was diagnosed in 37 patients (86.0\%) 3 months after enrollment. Our patients predominantly experienced sensory symptoms, followed by autonomic symptoms. No patients reported motor symptoms or weakness. Longitudinal NCS demonstrated reduced NCV and amplitudes subsequent to taxane treatment compared with those measured before chemotherapy, although only three (8.1\%) patients had sural sensory nerve action potential amplitudes outside the normal range.

The reported prevalence of TIN varies, ranging from 57 to $83 \%$ for paclitaxel and 11 to $64 \%$ for docetaxel..$^{13}$ We diagnosed 37 patients (86.0\%) with TIN, which is in accordance

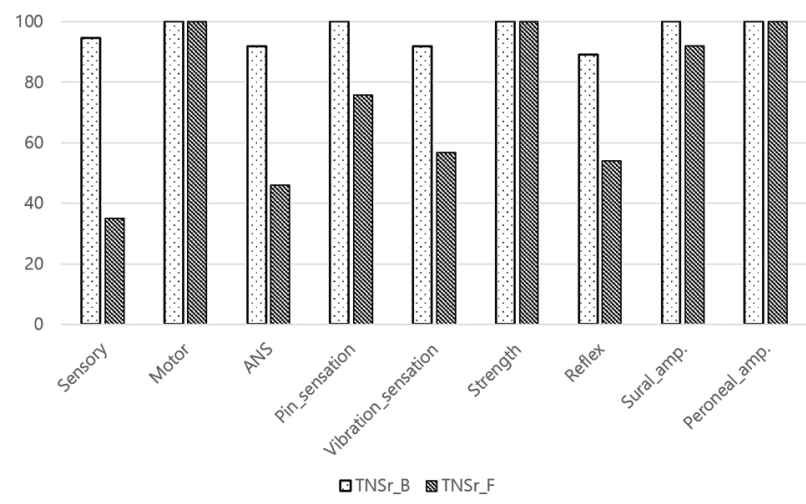

Fig. 1 Percentage of patients with normal Total Neuropathy Score (TNSr) before and after chemotherapy. Sensory and autonomic symptoms were the most frequent abnormalities after chemotherapy, followed by abnormal tendon reflex and vibration sensibility. Abbreviations: Amp., amplitude; TNSr_B, baseline TNSr; TNSr_F, 3-month TNSr. with previous studies. Several risk factors for TIN were identified, including health status, obesity, age, the cumulative dose of chemotherapeutic agents, treatment schedule, and infusion time. ${ }^{14-16}$ In this study, no significant differences in clinical features-obesity, age, the cumulative doses of taxane, and treatment interval (weekly vs. once every 3 week) were observed between the TIN and no TIN groups. These results might be originated from the small sample size and further study with larger number of patients will give more epidemiologic information of TIN.

TIN typically presents as distal sensory neuropathy and could persist, after chemotherapy is completed ${ }^{17}$ In the present study, all patients with TIN had sensory neuropathy without motor impairment, which contradicts a recent study that observed considerable motor neuropathy in patients with TIN. $^{3}$ They used NCI-CTCAE which determine the neuropathy status by clinician's decision. We assessed motor function by physical examination and patient-reported symptoms by TNSr; hence, our results emphasize the importance of a combination of objective examination and patient-reported symptoms for the evaluation of CIPN. However, we could not preclude the possibility of bias from the small sample size and further study with larger number of patients diagnosed by serial TNSr is needed.

In this study, the frequency of sensory symptoms measured by the EORTC-CIPN20 was higher than that as assessed by the TNSr, and the TNSr sensory symptom and pin sensation scores positively correlated with the EORTC-CIPN20 sensory symptom scores. Patient-reported outcome measures, such as the EORTC-CIPN20, are reported to be more sensitive than clinician-based instruments, and their correlation to objective neuropathy measures in CIPN studies has been validated. ${ }^{14,15}$ However these measures often overestimate CIPN because they do not evaluate objective signs of neuropathy. A recent Delphi survey rated brief patient-reported outcome questionnaires as having the highest clinical utility of available assessment tools and the TNSc, which includes the symptoms and clinical examination items of the TNS, as the best clinical/composite assessment tool. ${ }^{12}$ Another study demonstrated that brief patient-reported outcome questionnaires provide useful complementary information to the NCI-CTCAE scale. ${ }^{18}$ Because the TNS positively correlated with a patient-reported questionnaire in this study and is a composite scale of subjective symptoms and physical examination, it could be a useful sole screening tool for evaluating CIPN.

It remains unclear how taxanes induce TIN. It is postulated that taxanes interrupt axonal transport and damage the mitochondria, which causes axonal damage. ${ }^{17}$ It has been suggested that, among all the peripheral nerves, large myelinated sensory nerve fibers are the most susceptible to TIN. ${ }^{19,20}$ The results of NCS after taxane treatment also demonstrated taxane-induced axonal damage. However, TIN is characterized by sensory loss, neuropathic pain, and, rarely, motor impairment in the literature. ${ }^{16}$ Pure sensory neuropathy presenting as neuropathic pain without motor impairment is a typical feature of small fiber neuropathy, and evidence that taxanes damage small nerve fibers first 
Table 3 Correlation coefficients between TNSr and CIPN20 in taxane-induced neuropathy patients

\begin{tabular}{|l|l|l|l|l|l|}
\hline TNSr_F CIPN20_F & Sensory-hand & Sensory-foot & Motor & ANS & Total score \\
\hline Sensory & $0.645^{\mathrm{a}}(<0.001)$ & $\begin{array}{l}0.632^{\mathrm{a}} \\
(<0.001)\end{array}$ & $0.303(0.110)$ & $0.142(0.462)$ & $0.639^{\mathrm{a}}(<0.001)$ \\
\hline ANS & $-0.147(0.448)$ & $-0.172(0.374)$ & $0.285(0.134)$ & $\begin{array}{l}0.398^{\mathrm{b}} \\
(0.032)\end{array}$ & $0.025(0.898)$ \\
\hline Pin sensation & $0.380^{\mathrm{b}}(0.042)$ & $0.423^{\mathrm{b}}(0.022)$ & $0.317(0.094)$ & $0.126(0.516)$ & $0.428^{\mathrm{b}}(0.021)$ \\
\hline Vibration sensation & $-0.017(0.931)$ & $0.117(0.545)$ & $0.245(0.200)$ & $0.007(0.971)$ & $0.106(0.586)$ \\
\hline Total score & $0.369^{\mathrm{a}}(0.049)$ & $0.314(0.097)$ & $0.425^{\mathrm{a}}(0.022)$ & $0.324(0.087)$ & $0.470^{\mathrm{a}}(0.010)$ \\
\hline
\end{tabular}

Abbreviations: ANS, autonomic nervous system symptoms; TNSr_F, 3-month TNSr; CIPN20_F, 3-month CIPN20; sensory-hand, sensory symptoms of the hand as assessed by the CIPN20; sensory-foot, sensory symptoms of the foot as assessed by the CIPN20; sensory, sensory symptoms as assessed by TNSr.

Values are expressed as "correlation coefficient r ( $p$-value)".

aDenotes high significance $(p<.001)$.

benotes significance $(p<.05)$.

Table 4 Comparison of nerve conduction studies before and after chemotherapy in patients with taxane-induced neuropathy

\begin{tabular}{|l|l|l|l|}
\hline & Before CTx & After CTx & $p$-Value \\
\hline Peroneal motor amplitude $(\mathrm{mV})$ & $6.9 \pm 2.2$ & $5.8 \pm 1.7$ & $<0.001$ \\
\hline NCV $(\mathrm{m} / \mathrm{sec})$ & $50.6 \pm 3.4$ & $48.8 \pm 3.1$ & $<0.001$ \\
\hline Sural sensory amplitude $(\mu \mathrm{V})$ & $27.1 \pm 12.5$ & $20.8 \pm 10.0$ & $<0.001$ \\
\hline NCV $(\mathrm{m} / \mathrm{sec})$ & $45.9 \pm 3.9$ & $42.8 \pm 8.4$ & 0.046 \\
\hline Median sensory amplitude $(\mu \mathrm{V})$ & $57.2 \pm 19.1$ & $43.9 \pm 13.4$ & 0.004 \\
\hline NCV (m/sec) & $47.5 \pm 4.3$ & $46.1 \pm 5.0$ & $<0.001$ \\
\hline Ulnar sensory amplitude $(\mu \mathrm{V})$ & $35.2 \pm 12.9$ & $28.8 \pm 11.9$ & 0.001 \\
\hline NCV (m/sec) & $49.4 \pm 2.9$ & $4.7 \pm 3.1$ & $<0.001$ \\
\hline
\end{tabular}

Abbreviations: CTx, chemotherapy; NCV, nerve conduction velocity.

Values are expressed as "mean \pm SD".

has been reported. A study using a zebrafish model suggested that paclitaxel-induced neuropathy may depend on the interaction between the skin nerve endings and epidermal basal keratinocytes..$^{21}$ Another study showed a reduction of epidermal nerve fibers in the area of pain in TIN. ${ }^{13}$ We found that sensory symptoms were the most frequent symptoms followed by autonomic symptoms without motor impairment and that NCS values after chemotherapy were mostly within normal limits in patients with TIN. Therefore, we suggest that more specialized, objective tests for small nerve fibers, such as QST for heat stimulus and epidermal nerve fiber quantification, are needed to diagnose TIN in early stages.

Furthermore, we found that nine patients (20.9\%) had symptoms or signs of neuropathy before chemotherapy. Many studies on CIPN are of cross-sectional design, and longitudinal studies are uncommon in the literature. ${ }^{22}$ Molassiotis et al found that a considerable number of patients reported CIPN symptoms before chemotherapy and insisted that baseline assessment and longitudinal studies are essential for the identification of CIPN. ${ }^{3}$ Our results corroborate that a longitudinal assessment before and after chemotherapy is important for the accurate diagnosis of CIPN.

This study has several limitations. First, this was an observational study without controls; therefore, we were unable to calculate the sensitivity or specificity of the TNSr as a diagnostic tool for TIN. However, positive correlation of the TNSr with the EORTC-CIPN20 provided the validity of the TNSr to evaluate subjective symptoms. Diagnostic accuracy of TNS compared with other clinician-assessing scales have been validated previously. ${ }^{11,12}$ Therefore, we demonstrated the usefulness of this scale for the evaluation of CIPN, although we could not identify the diagnostic accuracy of the TNSr, Secondly, the sample size was too small to identify risk factors and epidemiologic data of TIN. Further studies with larger sample sizes are needed to fully evaluate putative risk factors.

\section{Conclusion}

TIN was primarily characterized as sensory neuropathy without motor impairment. A combination scale comprising a clinician-based scale and a patient-reported questionnaire and specialized tests for small nerve fibers should be considered as the proper assessment tools to evaluate TIN. Moreover, continual assessment from baseline with a composite scale is necessary for the accurate diagnosis of TIN because pre-existing symptoms are not uncommon in patients undergoing chemotherapy.

\section{Sources of Support}

This study was financially supported by faculty research abroad program of Chungnam National University in 2019. 
Conflict of Interest

None declared.

\section{References}

1 Zajaczkowska R, Kocot-Kepska M, Leppert W, Wrozosek A, Mika J, Wordliczek J. Mechanisms of chemotherapy-induced peripheral neuropathy. Int J Mol Sci 2019;20:1451

2 Park SB, Goldstein D, Krishnan AV, et al. Chemotherapy-induced peripheral neurotoxicity: a critical analysis. CA Cancer J Clin 2013;63(6):419-437

3 Molassiotis A, Cheng HL, Lopez V, et al. Are we mis-estimating chemotherapy-induced peripheral neuropathy? Analysis of assessment methodologies from a prospective, multinational, longitudinal cohort study of patients receiving neurotoxic chemotherapy. BMC Cancer 2019;19(1):132

4 Seretny $M$, Currie GL, Sena ES, et al. Incidence, prevalence, and predictors of chemotherapy-induced peripheral neuropathy: a systematic review and meta-analysis. Pain 2014;155(12):2461-2470

5 Trotti A, Colevas AD, Setser A, et al. CTCAE v3.0: development of a comprehensive grading system for the adverse effects of cancer treatment. Semin Radiat Oncol 2003;13(3):176-181

6 Cavaletti G, Frigeni B, Lanzani F, et al. Chemotherapy-induced peripheral neurotoxicity assessment: a critical revision of the currently available tools. Eur J Cancer 2010;46(3):479-494

7 Frigeni B, Piatti M, Lanzani F, et al. Chemotherapy-induced peripheral neurotoxicity can be misdiagnosed by the National Cancer Institute Common Toxicity scale. J Peripher Nerv Syst 2011;16(3):228-236

8 Postma TJ, Aaronson NK, Heimans JJ, et al. EORTC Quality of Life Group. The development of an EORTC quality of life questionnaire to assess chemotherapy-induced peripheral neuropathy: the QLQ-CIPN20. Eur J Cancer 2005;41(8):1135-1139

9 Lavoie Smith EM, Haupt R, Kelly JP, et al. The content validity of a chemotherapy-induced peripheral neuropathy patient-reported outcome Measure. Oncol Nurs Forum 2017;44(5):580-588

10 Cavaletti G, Frigeni B, Lanzani F, et al; Italian NETox Group. The Total Neuropathy Score as an assessment tool for grading the course of chemotherapy-induced peripheral neurotoxicity: comparison with the National Cancer Institute-Common Toxicity Scale. J Peripher Nerv Syst 2007;12(3):210-215

11 Binda D, Cavaletti G, Cornblath DR, Merkies IS; CI-PeriNomS study group. Rasch-Transformed Total Neuropathy Score clinical version $(\operatorname{RT}-\mathrm{TNSc}(\odot))$ in patients with chemotherapy-induced peripheral neuropathy. J Peripher Nerv Syst 2015;20(3):328-332

12 McCrary JM, Goldstein D, Boyle F, et al. IN FOCUS Delphi working party. Optimal clinical assessment strategies for chemotherapy-induced peripheral neuropathy (CIPN): a systematic review and Delphi survey. Support Care Cancer 2017;25(11):3485-3493

13 Krøigård T, Schrøder HD, Qvortrup C, et al. Characterization and diagnostic evaluation of chronic polyneuropathies induced by oxaliplatin and docetaxel comparing skin biopsy to quantitative sensory testing and nerve conduction studies. Eur J Neurol 2014;21(4):623-629

14 Monfort SM, Pan X, Patrick R, et al. Gait, balance, and patient-reported outcomes during taxane-based chemotherapy in early-stage breast cancer patients. Breast Cancer Res Treat 2017;164(1):69-77

15 Lavoie Smith EM, Barton DL, Qin R, Steen PD, Aaronson NK, Loprinzi CL. Assessing patient-reported peripheral neuropathy: the reliability and validity of the European Organization for Research and Treatment of Cancer QLQ-CIPN20 Questionnaire. Qual Life Res 2013;22(10):2787-2799

16 Kerckhove N, Collin A, Condé S, Chaleteix C, Pezet D, Balayssac D. Long-term effects, pathophysiological mechanisms, and risk factors of chemotherapy-induced peripheral neuropathies: a comprehensive literature review. Front Pharmacol 2017;8:86

17 Staff NP, Grisold A, Grisold W, Windebank AJ. Chemotherapy-induced peripheral neuropathy: a current review. Ann Neurol 2017;81(6):772-781

18 Tan AC, McCrary JM, Park SB, Trinh T, Goldstein D. Chemotherapy-induced peripheral neuropathy-patientreported outcomes compared with NCI-CTCAE grade. Support Care Cancer 2019;27(12):4771-4777

19 Argyriou AA, Koltzenburg M, Polychronopoulos P, Papapetropoulos S, Kalofonos HP. Peripheral nerve damage associated with administration of taxanes in patients with cancer. Crit Rev Oncol Hematol 2008;66(3):218-228

20 Carlson K, Ocean AJ. Peripheral neuropathy with microtubule-targeting agents: occurrence and management approach. Clin Breast Cancer 2011;11(2):73-81

21 Cavaletti G, Jann S, Pace A, et al. Italian NETox Group. Multi-center assessment of the Total Neuropathy Score for chemotherapy-induced peripheral neurotoxicity. J Peripher Nerv Syst 2006;11(2):135-141

22 Rivera DR, Ganz PA, Weyrich MS, Bandos H, Melnikow J. Chemotherapy-associated peripheral neuropathy in patients with early-stage breast cancer: a systematic review. J Natl Cancer Inst 2018;110(2):131-140 